Jing Huang*, Jin-Xia Ma, Ping Wei and Ji-Xing Zhao*

\title{
Crystal structure of bis $\left\{\mu_{2}\right.$-bis $\{((((1-$ methoxyimino)ethyl)phenyl)imino)methyl)-2- phenolato- $\mathrm{K}^{3} \mathrm{~N}, \mathrm{O}: \mathrm{O}$ \}copper(II)\}, $\mathrm{C}_{68} \mathrm{H}_{68} \mathrm{Cu}_{2} \mathrm{~N}_{8} \mathrm{O}_{8}$
}

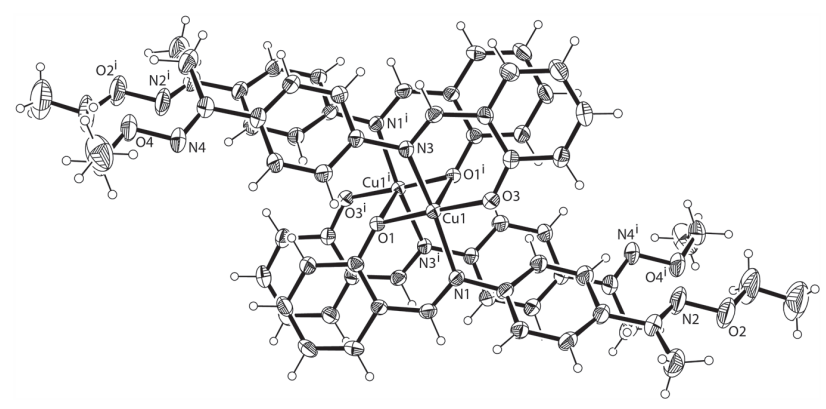

https://doi.org/10.1515/ncrs-2019-0062

Received January 20, 2019; accepted March 29, 2019; available online May 8, 2019

Table 1: Data collection and handling.

\begin{tabular}{ll}
\hline Crystal: & Dark brown block \\
Size: & $0.27 \times 0.26 \times 0.22 \mathrm{~mm}$ \\
Wavelength: & Mo $K \alpha$ radiation $(0.71073 \AA$ A $)$ \\
$\mu:$ & $0.76 \mathrm{~mm}^{-1}$ \\
Diffractometer, scan mode: & Bruker APEX-II, $\varphi$ and $\omega$-scans \\
$\theta_{\text {max }}$, completeness: & $27.6^{\circ},>99 \%$ \\
$N(h k l)_{\text {measured }}, N(h k l)_{\text {unique }}, R_{\text {int }}:$ & $20682,7046,0.061$ \\
Criterion for $I_{\text {obs }}, N\left(h k l l_{\mathrm{gt}}:\right.$ & $I_{\text {obs }}>2 \sigma\left(I_{\text {obs }}\right), 4271$ \\
$N(\text { param })_{\text {refined }}:$ & 392 \\
Programs: & Bruker programs [1], SHELX [2], \\
& OLEX2 [3] \\
\hline
\end{tabular}

(328.0 mg, $2 \mathrm{mmol}$ ) was added an ethanolic solution ( $5 \mathrm{~mL}$ ) of salicylaldehyde ( $244.1 \mathrm{mg}, 2 \mathrm{mmol})$. After stirring and refluxing for 9 hours at $328 \mathrm{~K}$, the mixture was filtered at room temperature. The product was dried under vacuum and a yellow solid was obtained (yield 67.3\%, m.p. 389-397 K). Elemental analysis: Anal. Calcd. For $\mathrm{C}_{17} \mathrm{H}_{18} \mathrm{~N}_{2} \mathrm{O}_{2}$ : C, 72.32\%; H, 6.43\%; N, 9.92\%; Found: C, 72.61\%; H, 6.71\%; N, 9.71\%.

Synthesis of the copper(II) complex: To an ethanolic solution (2 mL) of copper(II) acetate monohydrate $(2.0 \mathrm{mg}$, $10 \mathrm{mmol}$ ) was added an chloroform solution $(4 \mathrm{~mL})$ of ligand $(5.7 \mathrm{mg}, 20 \mathrm{mmol}$ ) at room temperature. The mixture was filtered after being stirred for $2 \mathrm{~h}$. Then the filtrate was allowed to stand for 12 days. Several clear dark brown crystals were obtained. Anal. Calcd. for $\mathrm{C}_{68} \mathrm{H}_{68} \mathrm{Cu}_{2} \mathrm{~N}_{8} \mathrm{O}_{8}$ : C, 65.21\%; H, 5.47\%; N, 8.95\%. Found: C, 65.45\%; H, 5.76\%; N, 8.72\%.

\section{Experimental details}

Hydrogen atoms were placed in their geometrically idealized positions and constrained to ride on their parent atoms.

*Corresponding authors: Jing Huang, School of Chemical Engineering, Northwest Minzu University, Lanzhou 730030, People's Republic of China; and Key Laboratory for Utility of Environment-Friendly Composite Materials and Biomass in Universities of Gansu Province, Lanzhou 730124, People's Republic of China, e-mail: shiyihuangjing@126.com; and Ji-Xing Zhao, Analysis and Testing Center, Shihezi University, Shihezi 832003, People's Republic of China, e-mail: zhaojixing_2016@126.com

Jin-Xia Ma and Ping Wei: School of Chemical and Biological Engineering, Lanzhou Jiaotong University, Lanzhou 730070, P.R. China

\section{Comment}

Schiff bases have been reported as an important class of ligands [7]. Their metal complexes have been widely studied [8-11]. Our research group has focus on the study of Schiff base and their complexes. Recently much attention is being focused on analytical reagents for the detection and determination of some metal ions [12, 13], specific properties $[14,15]$ and supramolecular architectures [16-18] etc.

This work is licensed under the Creative Commons Attribution 4.0 Public 
Table 2: Fractional atomic coordinates and isotropic or equivalent isotropic displacement parameters $\left(\AA^{2}\right)$.

\begin{tabular}{|c|c|c|c|c|}
\hline Atom & $x$ & $y$ & $z$ & $U_{\text {iso }} * / U_{\text {eq }}$ \\
\hline Cu1 & $0.50267(2)$ & 0.58933(3) & $0.58334(3)$ & $0.03836(13)$ \\
\hline N1 & $0.44797(13)$ & $0.71956(17)$ & $0.51555(16)$ & $0.0384(6)$ \\
\hline N2 & $0.07705(19)$ & $0.8166(3)$ & $0.5883(3)$ & $0.0961(13)$ \\
\hline N3 & $0.57240(13)$ & $0.48781(18)$ & $0.66722(17)$ & $0.0383(6)$ \\
\hline N4 & $0.95590(16)$ & $0.4472(3)$ & $0.6188(2)$ & $0.0697(9)$ \\
\hline 01 & $0.57367(11)$ & $0.56515(14)$ & $0.49471(14)$ & $0.0409(5)$ \\
\hline 02 & $0.00108(16)$ & $0.8596(3)$ & $0.6081(3)$ & $0.1143(13)$ \\
\hline 03 & $0.43190(12)$ & $0.61882(16)$ & $0.66898(15)$ & $0.0491(6)$ \\
\hline 04 & $1.03612(15)$ & $0.4091(2)$ & $0.6061(2)$ & $0.0849(9)$ \\
\hline $\mathrm{C} 1$ & $0.60360(17)$ & $0.6427(2)$ & $0.4473(2)$ & $0.0374(7)$ \\
\hline C2 & $0.67952(18)$ & $0.6268(2)$ & $0.4140(2)$ & $0.0491(8)$ \\
\hline $\mathrm{H} 2$ & 0.709734 & 0.563249 & 0.428737 & $0.059^{\star}$ \\
\hline C3 & $0.71048(19)$ & $0.7021(3)$ & $0.3604(2)$ & $0.0563(9)$ \\
\hline H3 & 0.760915 & 0.688749 & 0.339254 & $0.068^{*}$ \\
\hline C4 & $0.6676(2)$ & $0.7979(3)$ & $0.3373(2)$ & $0.0595(10)$ \\
\hline H4 & 0.688371 & 0.848643 & .300479 & $0.071^{*}$ \\
\hline C5 & $0.5943(2)$ & $0.8163(2)$ & $0.3698(2)$ & $0.0525(9)$ \\
\hline H5 & 0.565250 & 0.880479 & 0.354214 & $0.063^{\star}$ \\
\hline C6 & $0.56080(17)$ & $0.7424(2)$ & $0.4258(2)$ & $0.0390(7)$ \\
\hline $\mathrm{C7}$ & $0.48410(17)$ & $5(2)$ & $0(2)$ & $0.0425(7)$ \\
\hline H7 & 0.457126 & 0.832379 & 0.432589 & 0.051 * \\
\hline $\mathrm{C} 8$ & $0.37071(17)$ & $0.7648(2)$ & $0.5364(2)$ & $0.0417(7)$ \\
\hline C9 & $0.36063(18)$ & $0.8760(2)$ & $0.5518(2)$ & $0.0477(8)$ \\
\hline H9 & 0.404643 & 0.924257 & 0.548835 & $0.057^{\star}$ \\
\hline C10 & $0.28483(19)$ & $0.9151(2)$ & $0.5715(2)$ & $0.0540(9)$ \\
\hline $\mathrm{H} 10$ & 0.279098 & 0.989699 & 0.582439 & $0.065^{\star}$ \\
\hline C11 & $0.21759(19)$ & $0.8467(3)$ & $0.5755(2)$ & $0.0525(9)$ \\
\hline C12 & $0.22922(19)$ & $0.7352(3)$ & $0.5580(2)$ & $0.0586(10)$ \\
\hline H12 & 0.184701 & 0.687181 & 0.558600 & $0.070^{*}$ \\
\hline C13 & $0.30369(18)$ & $0.6947(2)$ & $0.5401(2)$ & $0.0516(9)$ \\
\hline H13 & 0.309651 & 0.619833 & 0.530264 & $0.062 *$ \\
\hline C14 & $0.1354(2)$ & $0.8868(3)$ & $0.5964(3)$ & $0.0658(11)$ \\
\hline C15 & $0.1242(3)$ & $1.0016(3)$ & $0.6272(3)$ & $0.0998(16)$ \\
\hline H15A & 0.126060 & 1.051828 & 0.579741 & $0.150^{*}$ \\
\hline H15B & 0.167777 & 1.019065 & 0.672048 & $0.150^{\star}$ \\
\hline $\mathrm{H} 15 \mathrm{C}$ & 0.071639 & 1.007782 & 0.649596 & $0.150^{*}$ \\
\hline C16 & $-0.0622(3)$ & $0.7776(4)$ & $0.5916(5)$ & $0.167(3)$ \\
\hline H16A & -0.062811 & 0.750697 & 0.532728 & $0.201^{*}$ \\
\hline H16B & -0.050368 & 0.716126 & 0.630769 & 0.201 * \\
\hline C17 & $-0.1400(3)$ & $0.8224(5)$ & $0.6032(5)$ & $0.182(4)$ \\
\hline H17A & -0.144886 & 0.829370 & 0.664008 & $0.273^{\star}$ \\
\hline H17B & -0.182932 & 0.774754 & 0.577155 & $0.273^{*}$ \\
\hline $\mathrm{H} 17 \mathrm{C}$ & -0.145467 & 0.893514 & 0.576384 & $0.273^{*}$ \\
\hline C18 & $0.33830(19)$ & $0.5704(3)$ & $0.7675(2)$ & $0.0497(8)$ \\
\hline H18 & 0.304439 & 0.629353 & 0.748822 & $0.060^{\star}$ \\
\hline C19 & $0.3157(2)$ & $0.5038(3)$ & $0.8322(2)$ & $0.0578(9)$ \\
\hline H19 & 0.267507 & 0.519038 & 0.857078 & 0.069 * \\
\hline $\mathrm{C} 20$ & $0.3638(2)$ & $0.4137(3)$ & $0.8611(3)$ & $0.0632(10)$ \\
\hline $\mathrm{H} 20$ & 0.347821 & 0.368299 & 0.904491 & $0.076^{*}$ \\
\hline C21 & $0.4353(2)$ & $0.3932(3)$ & $0.8245(2)$ & $0.0541(9)$ \\
\hline $\mathrm{H} 21$ & 0.467694 & 0.333079 & 0.843671 & $0.065^{\star}$ \\
\hline $\mathrm{C} 22$ & $0.46104(17)$ & $0.4604(2)$ & $0.7589(2)$ & $0.0410(7)$ \\
\hline $\mathrm{C} 23$ & $0.41170(17)$ & $0.5518(2)$ & $0.7287(2)$ & $0.0404(7)$ \\
\hline C24 & $0.53952(17)$ & $0.4397(2)$ & $0.7298(2)$ & $0.0424(8)$ \\
\hline $\mathrm{H} 24$ & 0.571236 & 0.384940 & 0.759136 & $0.051^{*}$ \\
\hline
\end{tabular}

Table 2 (continued)

\begin{tabular}{lrrrr}
\hline Atom & $\boldsymbol{x}$ & $\boldsymbol{y}$ & $\boldsymbol{z}$ & \multicolumn{1}{c}{$\boldsymbol{U}_{\text {iso }}{ }^{*} \boldsymbol{U}_{\text {eq }}$} \\
\hline C25 & $0.65633(17)$ & $0.4564(2)$ & $0.6575(2)$ & $0.0423(7)$ \\
C26 & $0.68336(18)$ & $0.3500(2)$ & $0.6717(2)$ & $0.0497(8)$ \\
H26 & 0.646361 & 0.296424 & 0.686094 & $0.060^{*}$ \\
C27 & $0.76483(18)$ & $0.3216(3)$ & $0.6648(2)$ & $0.0565(9)$ \\
H27 & 0.781884 & 0.249368 & 0.675173 & $0.068^{*}$ \\
C28 & $0.82126(18)$ & $0.3985(3)$ & $0.6427(2)$ & $0.0513(8)$ \\
C29 & $0.79328(18)$ & $0.5062(3)$ & $0.6282(2)$ & $0.0546(9)$ \\
H29 & 0.830210 & 0.559697 & 0.613516 & $0.066^{*}$ \\
C30 & $0.71214(18)$ & $0.5352(2)$ & $0.6351(2)$ & $0.0486(8)$ \\
H30 & 0.694841 & 0.607414 & 0.624875 & $0.058^{*}$ \\
C31 & $0.9087(2)$ & $0.3672(3)$ & $0.6327(3)$ & $0.0608(10)$ \\
C32 & $0.9354(2)$ & $0.2501(3)$ & $0.6379(4)$ & $0.1046(18)$ \\
H32A & 0.994038 & 0.245849 & 0.634987 & $0.157^{*}$ \\
H32B & 0.906886 & 0.209848 & 0.590383 & $0.157^{*}$ \\
H32C & 0.922447 & 0.218946 & 0.691569 & $0.157^{*}$ \\
C33 & $1.0885(2)$ & $0.5029(4)$ & $0.6069(3)$ & $0.0847(14)$ \\
H33A & 1.060748 & 0.560113 & 0.571086 & $0.102^{*}$ \\
H33B & 1.138918 & 0.483465 & 0.582446 & $0.102^{*}$ \\
C34 & $1.1097(3)$ & $0.5453(5)$ & $0.6953(4)$ & $0.127(2)$ \\
H34A & 1.059877 & 0.564256 & 0.719844 & $0.191^{*}$ \\
H34B & 1.143955 & 0.609420 & 0.693492 & $0.191^{*}$ \\
H34C & 1.139184 & 0.489854 & 0.730353 & $0.191^{*}$ \\
\hline
\end{tabular}

The single crystal structure of the title complex was determined by X-ray crystallography. This crystal structure of the title complex is built up by the $\mathrm{C}_{68} \mathrm{H}_{68} \mathrm{Cu}_{2} \mathrm{~N}_{8} \mathrm{O}_{8}$ complex ( $c f$. the figure). In the title complex, $\mathrm{Cu} 1$ atom is five-coordinate, with a $\mathrm{N}_{2} \mathrm{O}_{3}$ coordination environment with three $\mathrm{O}$ atoms $(\mathrm{O} 1$, $\mathrm{O} 3$ and $\mathrm{O}^{\mathrm{i}}$ ) and two $\mathrm{N}$ atoms ( $\mathrm{N} 1$ and $\mathrm{N} 3$ ). The $\mathrm{Cu} 1-\mathrm{O} 1$ bond length is 1.914(2) $\AA$ and the $\mathrm{Cu1}-\mathrm{O} 3$ is 1.886(2) $\AA$ and $\mathrm{Cu1}-\mathrm{O} 1^{\mathrm{i}}$ is $2.490 \AA$, respectively. The Cu1-N1 bond length is 2.049(2) $\AA$ and the Cu1-N3 is 2.044(2) $\AA$, respectively.

Geometric parameters are all in the expected ranges for such a complex.

\section{References}

1. Bruker. APEX2, SAINT and SADABS. Brucker AXS Inc., Madison, WI, USA (2012).

2. Sheldrick, G. M.: A short history of SHELX. Acta Crystallogr. A64 (2008) 112-122.

3. Dolomanov, O. V.; Bourhis, L. J.; Gildea, R. J.; Howard, J. A. K.; Puschmann, H.: OLEX2: a complete structure solution, refinement and analysis program. J. Appl. Cryst. 42 (2009) 339-341.

4. Han, Y.; Sun, J. J.; Wang, G. L.; Yan, C. G.: Synthesis and crystal structures of meso-substituted calix[4]pyrrole monoSchiff bases and transition metal complexes. J. Incl. Phenom. Macrocycl. Chem. 81 (2014) 215-224.

5. Li, P. P.; Ma, J. X.; Li, Q. L.; Zhao, J. X.; Zhao, L.: Crystal structure of (E)-1-(4-(((E)-2-hydroxy-5-methylbenzylidene)amino)phenyl) ethan-1-one 0-methyl oxime, $\mathrm{C}_{17} \mathrm{H}_{18} \mathrm{~N}_{2} \mathrm{O}_{2}$. Z. Kristallogr. NCS 233 (2018) 533-534. 
6. Gong, Y. L.; Lu, Y.; Ma, H. Y.; Ding, G.; Zhang, S. T.; Luo, Z. P.; Li, H. R.; Gao, F.: Efficient enhancement of internal proton transfer of branched $\pi$-extended organic chromophore under one-photon andnear-infrared two-photon irradiation. Chem. Phys. Lett. 619 (2015) 201-207.

7. Li, Q. L.; Li, P. P.; Ma, J. X.; Zhao, J. X.; Zhao, L.: Crystal structure of bis\{2-((E)-((4-((E)-1-(methoxyimino)ethyl)phenyl)imino) methyl)phenolato- $\mathrm{K}^{2} \mathrm{~N}, \mathrm{O}$ \}zinc(II), $\mathrm{C}_{32} \mathrm{H}_{30} \mathrm{~N}_{4} \mathrm{O}_{4} \mathrm{Zn}$. Z. Kristallogr. NCS 233 (2018) 637-639.

8. Sun, Y. X.; Lu, R. E.; Li, X. R.; Zhao, Y. Y.; Li, C. Y.: A Schiff base ligand containing oxime group and its $\mathrm{Cu}(\mathrm{II})$ complex: Syntheses and supramolecular structures. Chin. J. Inorg. Chem. 31 (2015) 1055-1062.

9. Zhao, L.; An, Q. Q.; Zhao, J. X.; Li, P. P.; Wang, F.: Crystal structure of bis(2-((E)-((4-((E)-1-(ethoxyimino)ethyl)phenyl)imino)

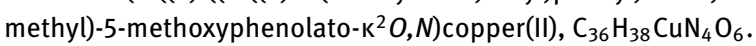
Z. Kristallogr. NCS 232 (2017) 595-597.

10. Zhao, J. X.; Zhao, L.; Li, P. P.; Zhu, Z. B.: Crystal structure of bis $\{5-$ methoxy-2-((E)-((4-((E)-1-(methoxyimino)ethyl)phenyl) imino)methyl)phenolato- $\left.\left.\mathrm{K}^{2} \mathrm{~N}, \mathrm{O}\right)\right\} \operatorname{copper}(\mathrm{II}), \mathrm{C}_{34} \mathrm{H}_{34} \mathrm{CuN}_{4} \mathrm{O}_{6}$. Z. Kristallogr. NCS 232 (2017) 863-865.

11. Li, P. P.; Li, Q. L.; Ma, J. X.; Zhao, J. X.; Zhao, L.: Crystal structure of bis\{4-methyl-2-((E)-((4-((E)-1-(ethoxyimino)ethyl)phenyl)

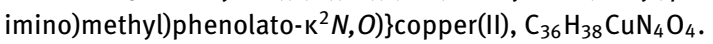
Z. Kristallogr. NCS 233 (2018) 763-765.

12. Dong, Y. J.; Li, X. L.; Zhang, Y.; Dong, W. K.: A highly selective visual and fluorescent sensor for $\mathrm{Pb}^{2+}$ and $\mathrm{Zn}^{2+}$ and crystal structure of $\mathrm{Cu}^{2+}$ complex based-on a novel single-armed Salamotype bisoxime. Supramol. Chem. 29 (2017) 518-527.
13. Dong, W. K.; Li, X. L.; Wang, L.; Zhang, Y.; Ding, Y. J.: A new application of Salamo-type bisoximes: as a relay-sensor for $\mathrm{Zn}^{2+} / \mathrm{Cu}^{2+}$ and its novel complexes for successive sensing of $\mathrm{H}^{+} / \mathrm{OH}^{-}$. Sens. Actuators B229 (2016) 370-378.

14. Handa, M.; Kanagawa, K.; Yano, N.; Yairi, H.; Okuno, A.; Mitsumi, M.; Kataoka, Y.: Synthesis and properties of a trinuclear copper(II) complex of a ligand with phthalocyanine and Schiff-base coordination sites. J. Porphyrins Phthalocyanines 22 (2018) 1-7.

15. Sun, Y. X.; Li, C. Y.; Yang, C. J.; Zhao, Y. Y.; Guo, J. Q.; Yu, B.: Two $\mathrm{Cu}(I I)$ complexes with Schiff base ligands: Syntheses, crystal structure, spectroscopic roperties, and substituent effect. Chin. J. Inorg. Chem. 32 (2016) 327-335.

16. Zaltariov, M. F.; Cazacu, M.; Avadanei, M.; Shova, S.; Balan, M.; Vornicu, N.; Vlad, A.; Dobrov, A.; Varganici, C. D.: Synthesis, characterization and antimicrobial activity of new $\mathrm{Cu}(\mathrm{II})$ and $\mathrm{Zn}$ (II) complexes with Schiff bases derived from trimethylsilyl-propyl-p-aminobenzoate. Polyhedron 100 (2015) 121-131.

17. Zhao, L.; Dong, X. T.; Cheng, Q.; Zhao, J. X.; Wang, L.: Synthesis, crystal structure and spectral properties of a $2 D$ supramolecular copper(II) complex with 1-(4-\{[(E)-3-ethoxyl2-hydroxybenzylidene]amino\}phenyl)ethanone oxime. Synth. React. Inorg. Met.-Org. Nano-Met. Chem. 43 (2013) 1241-1246.

18. Sun, Y. X.; Zhao, Y. Y.; Li, C. Y.; Yu, B.; Guo, J. Q.; Li, J.: Supramolecular cobalt(II) and copper(II) complexes with Schiff base ligand: Syntheses, characterizations and crystal structures. Chin. J. Inorg. Chem. 32 (2016) 913-920. 